\title{
CHALLENGES FOR GENERIC DRUG PRODUCERS
}

\section{Dear Reader,}

In the fiercely competitive global market for generic drugs, the complexities and interconnectivities come to the fore when a drug is recalled. In recent months, as you may be aware, the sartans (also known as AT1 receptor antagonists, angiotensin II receptor blockers/antagonists) have come into the limelight due to the recall of some of them from the regulated markets of USA and Europe.

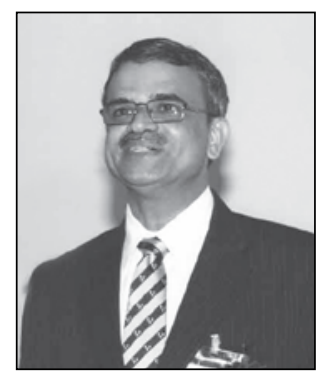

Since the introduction of losartan in the mid-nineties of the last century, several other sartans, including valsartan, candesartan, irbesartan and olmesartan, have been developed and enjoy great popularity in anti-hypertensive drug therapy, often in combination with other drugs. Now largely off-patent, the manufacturing of these bulk drugs and their formulations have been taken over by generic players from China, India and a few other countries. Following the detection of the carcinogenic impurity $N$-nitroso-dimethylamine (NDMA) in valsartan manufactured by Zhejiang Huahai Pharmaceutical to the extent of about60 ppm in formulations, all NDMA-impurity-containing sartans have been withdrawn from these markets, beginning July 2018.

While NDMA is formed in the synthetic process used by Zhejiang Huahai, it is not formed in the original manufacturing process developed by Ciba-Geigy. The NDMA formation is connected with the synthesis of the tetrazole ring structure, which is found in valsartan, candesartan, irbesartan, losartan and olmesartan. The US-FDA in St. Louis, Missouri has developed a head-space GC/MS method for the detection and estimation of NDMA.

Very recently, $N$-nitroso-diethylamine (NDEA) has been detected in valsartan and irbesartan exported from India. In one case, no NDMA had been detected earlier in its valsartan, but now NDEA has been detected in its batches.

No immediate risk is forseen due to the impurity level present and the average daily intake. An abrupt discontinuation of anti-hypertensive drug therapy with the tainted sartans is riskier and must be carried out, if it becomes necessary, under medical supervision and guidance.

The above recalls serve as another warning signal to the policy makers in India and those Indian manufacturers of bulk drugs pharmaceuticals who are highly dependent on imports of intermediates or APls from Chinese companies.

Of course, where there are challenges, there are also opportunities waiting to be grabbed.

Of the group of about 23 drugs which have been introduced in a European country in recent months, five of them are non-chiral chemical entities (tivozanib, glycerol phenyl butyrate, 
cariprazin, methacetin and patiromer) and seven of them are chiral chemical entities (telotristatethyl, sonidegib, letermovir, ertugliflozin, padeliporfin, desfesoterodin and bictegravir). Eleven of them are biologicals (avelumab, genetically modified T-cell preparation, ocrelizumab, guselkumab, bezlotoxumab, burosumab, vaccine against Herpes zoster, rurioctog alfa pegol, benralizumab, emicizumab immunoglobulin and velmanase alpha enzyme) with monoclonal antibodies dominating the scenario. The above list is non-exhaustive, but it tells you loud and clear in which way pharmaceutical research and development is heading.

Happy reading!

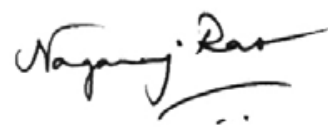

Dr. Nagaraj N. Rao Associate Editor Indian Drugs

\section{About The Associate Editor}

Dr. Nagaraj Narayan Rao obtained Bachelor's degrees in Science (Chemistry) and in the Technology of Pharmaceuticals and Fine Chemicals from the University of Mumbai. After working with Colgate-Palmolive (India) for two years as a laboratory chemist, he obtained his doctorate in science with magna cum laude from the University of Tuebingen, Germany, under the guidance of Prof. Dr. H. J. Roth. He carried out post-doctoral research at the Institute of Biotechnology of the Research Center Juelich, Germany. He was a member of the Editorial Board for the first official German-language version of the European Pharmacopoea. He was a visiting scientist at Juelich and a visiting faculty at the Institute of Chemical Technology Mumbai from 1993 to 2007 in the field of bioprocess technology. He has authored several original research articles, a patent, review articles and book chapters in the fields of pharmaceuticals, biotechnology, brewery and surface coatings. He was Chief Editor of the "Transactions of the MFAl" for a few years. He contributes a monthly 'Report from India' to a leading German technical journal since twelve years and is a distinguished alumnus of the Research Center Juelich.

Dr. Rao is co-founder of the RRR group of small and medium enterprises, manufacturing organic fine chemicals, formulations for surface coating technologies and fertilizers, process sensors and process units for life sciences, brewery and chemical process industries, as well as representing select overseas pharmaceutical and chemical equipment companies in India.

म 